\title{
Glucagonoma syndrome: A case report
}

\author{
JISHU WEI $^{1 *}$, SHIBO LIN $^{1 *}$, CONG WANG $^{2}$, JUNLI WU $^{1}$, ZHUYIN QIAN $^{1}$, \\ CUNCAI DAI $^{1}$, KUIRONG JIANG ${ }^{1}$ and YI MIAO ${ }^{1}$ \\ ${ }^{1}$ The Pancreas Center of Nanjing Medical University; ${ }^{2}$ Department of Pathology, \\ The First Affiliated Hospital of Nanjing Medical University, Nanjing, Jiangsu 210029, P.R. China
}

Received July 17, 2014; Accepted April 29, 2015

DOI: $10.3892 / \mathrm{ol} .2015 .3275$

\begin{abstract}
Necrolytic migratory erythema (NME), diabetes mellitus and glucagon-secreting tumors form the hallmarks of glucagonoma syndrome, and represent the major clinical manifestations of glucagonoma. NME is usually presented as the initial complaint of patients. Due to the rare incidence of glucagonoma, its diagnosis is often delayed, which leads to its progression. Here, we report a case of NME with a typical skin rash, which was misdiagnosed and treated with corticosteroids for two years. Removal of the tumor in the pancreatic body led to the rapid relief of the symptoms. The aim of the present study is to demonstrate the typical characteristics of glucagonoma syndrome to clinicians in order to improve its diagnosis and treatment.
\end{abstract}

\section{Introduction}

Glucagonoma is an uncommon neoplasm of the pancreatic neuroendocrine islet $\alpha$-cells, whereby the islet cells secrete abundant glucagon (1). The estimated annual incidence of glucagonoma is $\sim 1$ case per $20,000,000$ individuals (2). Due to the rarity of the disease, mortality rates remain unclear. In a study of 21 glucagonoma patients, Wermers et al (3) reported that nine patients succumbed to the diease after a mean duration of 4.91 years following diagnosis (3). Complete tumor resection may cure glucagonoma, with a 10 -year survival rate of $64.3 \%$ following surgery, however, liver and lymph node metastasis are major risk factors that contribute to tumor-associated mortality (4). For patients with unresectable advanced disease, tumor debulking, chemotherapy or somatostatin may also be considered to reduce the tumor-associated symptoms, however, the survival benefit of such treatments is limited $(2,3)$.

Correspondence to: Professor Yi Miao, The Pancreas Center of Nanjing Medical University, 300 Guangzhou Road, Nanjing, Jiangsu 210029, P.R. China

E-mail: miaoyi@njmu.edu.cn

*Contributed equally

Key words: necrolytic migratory erythema, diabetes mellitus, glucagonoma
Glucagonoma induces various manifestations, which are characterized by necrolytic migratory erythema (NME), diabetes mellitus (DM), weight loss, anemia and neuropsychiatric disturbances (1). NME represents the most specific manifestation of glucagonoma syndrome and has therefore provided the most valuable indications for diagnosis in the majority of previous cases (5). Early recognition of NME is likely to lead to a more rapid diagnosis of glucagonoma, thereby allowing surgical resection and achieving a promising therapeutic outcome. This study presents the case of a glucagonoma patient who recovered following spleen-preserving distal pancreatectomy.

\section{Case report}

In November 2010, a 50-year-old female presented to The Pancreas Center of Nanjing Medical University (Nanjing, China) with a 2-year history of a pruritic and ulcerating skin rash, which initially appeared at the waist and slowly progressed to the entire body within three months (Fig. 1A). Phacoscotasmus, cheilitis and glossitis were also present (Fig. 1B). The patient also complained of blurred vision and weight loss of $5 \mathrm{~kg}$. The patient was initially diagnosed with pemphigus and treated with prednisone without relief of the skin rash prior to her admission to our clinic. Physical examination revealed that the skin lesions were erythematous macules with erosions, serous exudate and crusting (Fig. 1C). A skin biopsy revealed that the skin layers were arranged normally with mild skin keratosis, basal pigmentation and perivascular infiltration of inflammatory cells in the superficial skin (Fig. 1D). Laboratory tests indicated elevated blood glucose (maximum $18 \mathrm{mmol} / \mathrm{l}$ ), anemia $(87 \mathrm{~g} / \mathrm{l})$ and hypoproteinemia (24.6 g/l). Following admission, the patient underwent abdominal computed tomography (CT) scanning, which disclosed a pancreatic body mass with low density (Fig. 2A). Given her history of the skin rash, DM and the pancreatic mass, the patient's blood glucagon concentration was measured, and her fasting glucagon level was noted to be elevated, at $1132.20 \mathrm{pg} / \mathrm{ml}$ (normal, $0-80 \mathrm{pg} / \mathrm{ml}$ ). The patient was first treated with octreotide and intravenous amino acid infusion. After the patient's nutrition was improved, spleen-preserving distal pancreatectomy was performed (Fig. 2B and C). The skin lesions were partially relieved following treatment with octreotide and intravenous amino acid infusion, and gradually recovered following surgery 
A
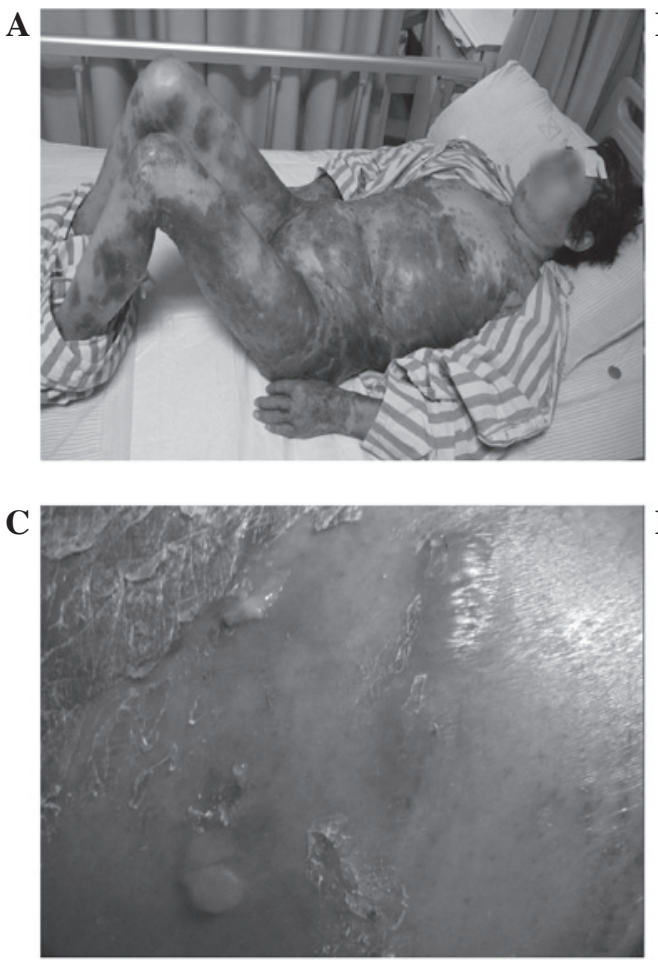

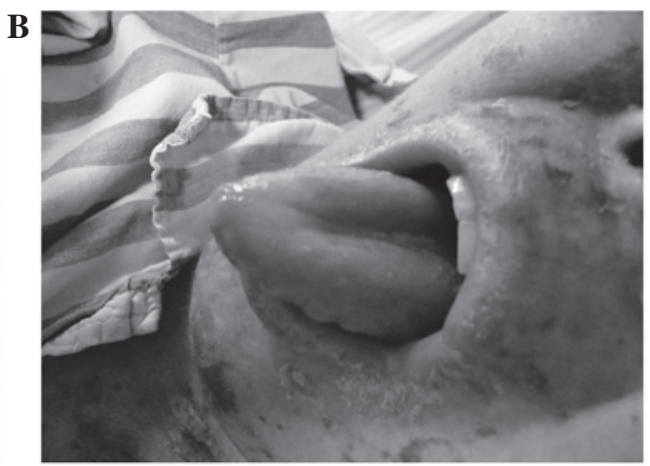

D

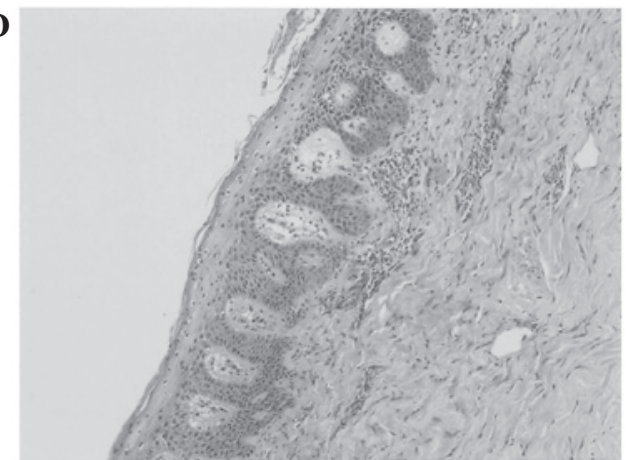

Figure 1. Skin lesions of glucagonoma. (A) Necrolytic migratory erythema. (B) Cheilitis and glossitis. (C) Skin lesions with erosions, serous exudate and crusting. (D) Pathology of the skin biopsy, observed with hematoxylin and eosin staining (magnification, x100).
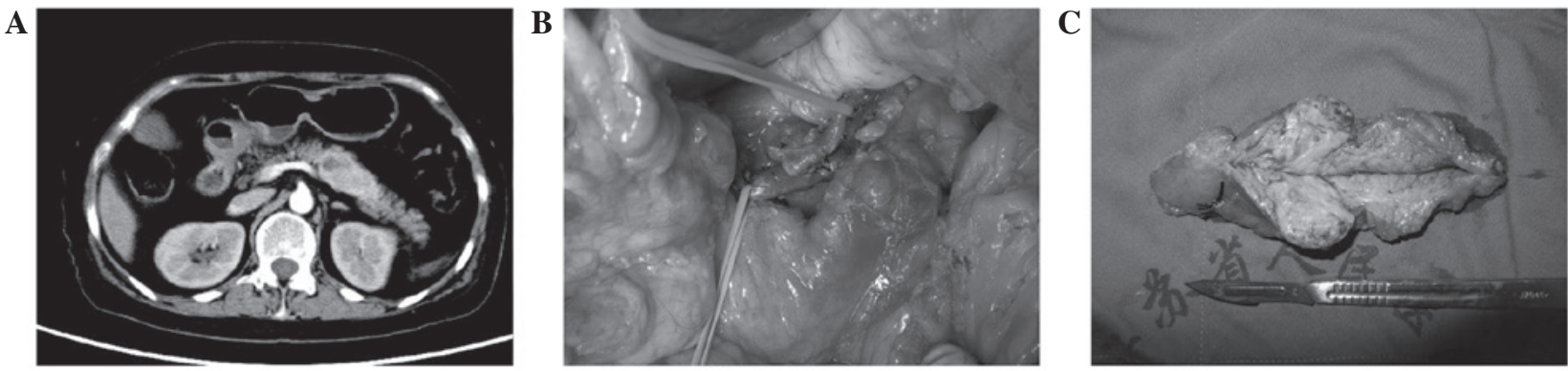

Figure 2. Tumor mass identified by computed tomography (CT) scan and surgical resection. (A) CT scanning indicated the pancreatic body tumor. (B) Laparotomy confirmed the tumor in the pancreatic body. (C) Resected tumor.
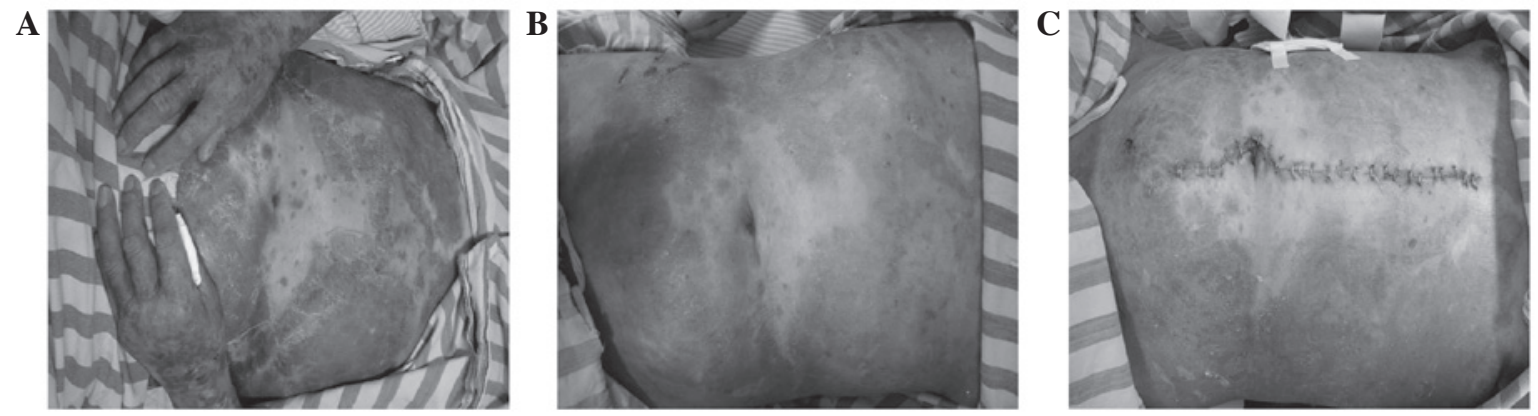

Figure 3. Changes in skin lesions following treatment. (A) Skin lesion upon admission. (B) Changes in skin lesions following treatment of octreotide and intravenous amino acid infusion. (C) Relief of skin lesion one week after surgery.

(Fig. 3A-C). Pathological examination confirmed the diagnosis of glucagonoma (Fig. 4A) and immunohistochemical staining revealed the positive staining of chromogranin and synaptophysin (Fig. 4B and C). Four weeks subsequent to discharge, the skin lesions were completely resolved and blood glucose returned to normal. At 3-year follow-up, the patient 

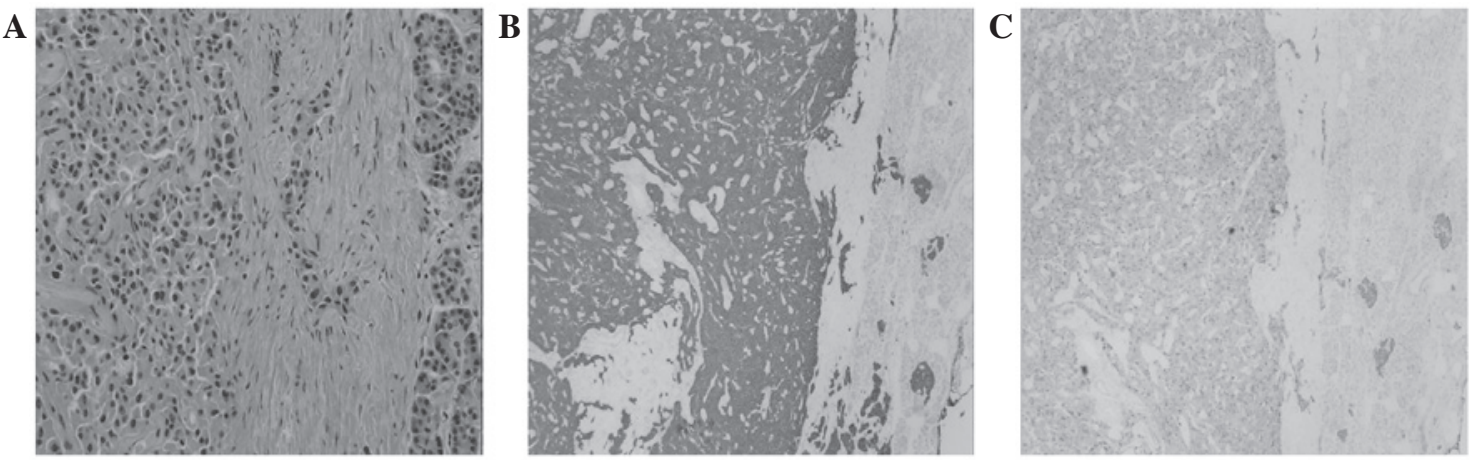

Figure 4. Pathological and immunohistochemistry analysis of glucagonoma. (A) Pathological analysis of tumor by hematoxyling and eosin staining (magnification, x100). (B) Positive staining of synaptophysin and (C) chromogranin by immunohistochemistry (magnification, $x 40$ ).

remained asymptomatic and no signs of recurrence were detected. The patient provided written informed consent prior to the publication of the study, and the study was approved by the research ethics committee of The First Affiliated Hospital of Nanjing Medical University (Nanjing, China).

\section{Discussion}

Glucagonoma, which accounts for $2 \%$ of islet cell carcinomas, is a rare neuroendocrine pancreatic tumor with an estimated incidence of one in 20 million (6). Although the diagnostic criteria for glucagonoma has already been established by Stacpoole (7), its rare incidence has hampered prompt diagnosis when the glucagonoma syndrome appears. To date, there are fewer than 300 cases reported in the literature and the largest case cohort included only 21 patients. Delayed diagnosis remains a major issue in the treatment of glucagonoma. The median time between the onset of glucagonoma syndrome and diagnosis is 3-4 years $(3,5)$.

Oversecretion of glucagon by islet $\alpha$-cells in tumors contributes to the paraneoplastic phenomenon, namely glucagonoma syndrome (5). Glucagon exhibits its physiological functions by increasing the hepatic glucose output and maintaining the blood glucose level (8). Glucagon also exerts a catabolic role by attenuating protein synthesis (9). The elevated glucagon secreted by glucagonoma results in amino acid catabolism and serum glucose elevation, which are considered to be responsible for skin lesions and DM (10). Glucagonoma syndrome consists of a triad comprising glucagon-secreting tumors, DM and NME (11).

NME is considered to be the hallmark of glucagonoma syndrome and is characterized by an annular pattern of erythema with centrally formed fragile vesicles, bullae and crusts (2). It is present in $\sim 65-70 \%$ of glucagonoma cases at the point of diagnosis (12). Although a skin biopsy of NME offers limited indications for the pathological diagnosis of glucagonoma, its early recognition may lead to further CT scanning and establish the diagnosis (13). It is worthy to note that celiac disease, malabsorption, cirrhosis, malignancy and pancreatitis may also present NME-like skin lesions, known as pseudoglucagonoma syndrome (14). In the present case, the NME had been misdiagnosed as immune pemphigus and treated with corticosteroids for two years. Although the corticosteroids treatments occasionally relieved the NME, persistent elevated glucagon inevitably induced its recurrence. In clinical practice, glucagonoma induced by NME should be considered if the skin lesions remain following conventional treatments.

DM is a further clinical hallmark of glucagonoma. Elevated glucagon levels promote the glucose output and antagonize the effect of insulin. Although only $40 \%$ of glucagonoma patients presented DM at the onset of symptoms, $\sim 90 \%$ went on to develop it (2). The severity of the diabetes remains controversial in the literature, and NME usually occurs prior to the emergence of the diabetes. In the present case, the patient exhibited intermittent glucose elevation one and a half years after the initiation of the skin rash, and the diabetes resulted in the rapid damage of her lens.

Glucagonoma is a slow-growing and relatively low-malignancy tumor. Although numerous glucagonoma patients suffer due to a delayed diagnosis, the majority still benefit from tumor resection. Metastasis represents the main prognostic factor for glucagonoma. Half of all glucagonomas are metastatic at the time of diagnosis (15). Patients without metastasis achieve a 10 -year survival rate of almost $100 \%$, compared with $51.6 \%$ of patients with metastasis (4). Synchronous resection of liver metastasis or liver transplantation provides a favorable outcome $(16,17)$. Whether patients would benefit from tumor debulking or chemotherapy remains to be elucidated $(5,17)$. For localized glucagonoma, removal of the tumor led to a notable improvement of the glucagonoma syndrome within several days of surgery (18). In the case of malnutrition, total parenteral nutrition with amino acid and caloric supplementation may be used to counteract the catabolic effects of high glucagon and reduce perioperative complications (2). Moreover, somatostatin analog may be useful in relieving glucagonoma syndrome by inhibiting glucagon secretion or counteracting its effect (19).

Although serum glucagon measurement and CT scanning are capable of establishing diagnosis in most cases, the bridge from glucagonoma syndrome to serum glucagon measurement and CT scanning remains a major obstacle to early diagnosis. Few clinicians suspect glucagonoma in its early stages due to its rare incidence. Numerous patients suffer due to the delayed diagnosis and may even miss out on the opportunity of curable resection. For this rare tumor, optimization of the diagnostic procedure based on glucagonoma syndrome is urgently needed. 


\section{Acknowledgements}

This study was supported by the Research Special Fund for Public Welfare of Health of China (201202007) and NSFC (81272239).

\section{References}

1. Tseng HC, Liu CT, Ho JC and Lin SH: Necrolytic migratory erythema and glucagonoma rising from pancreatic head. Pancreatology 13: 455-457, 2013.

2. Chastain MA: The glucagonoma syndrome: a review of its features and discussion of new perspectives. Am J Med Sci 321: 306-320, 2001.

3. Wermers RA, Fatourechi V, Wynne AG, Kvols LK and Lloyd RV: The glucagonoma syndrome. Clinical and pathologic features in 21 patients. Medicine (Baltimore) 75: 53-63, 1996.

4. Soga J and Yakuwa Y: Glucagonomas/diabetico-dermatogenic syndrome (DDS): a statistical evaluation of 407 reported cases. J Hepatobiliary Pancreat Surg 5: 312-319, 1998.

5. Eldor R, Glaser B, Fraenkel M, Doviner V, Salmon A and Gross DJ: Glucagonoma and the glucagonoma syndrome cumulative experience with an elusive endocrine tumour. Clin Endocrinol (Oxf) 74: 593-598, 2011.

6. Lo CH, Ho CL and Shih YL: Glucagonoma with necrolytic migratory erythema exhibiting responsiveness to subcutaneous octreotide injections. QJM 107: 157-158, 2014.

7. Stacpoole PW: The glucagonoma syndrome: clinical features, diagnosis and treatment. Endocr Rev 2: 347-361, 1981

8. Lefèbvre PJ: Glucagon and its family revisited. Diabetes Care 18: 715-730, 1995.

9. Charlton MR, Adey DB and Nair KS: Evidence for a catabolic role of glucagon during an amino acid load. J Clin Invest 98: 90-99, 1996.
10. van Beek AP, de Haas ER, van Vloten WA, Lips CJ, Roijers JF and Canninga-van Dijk MR: The glucagonoma syndrome and necrolytic migratory erythema: a clinical review. Eur J Endocrinol 151: 531-537, 2004

11. Shi W, Liao W, Mei X, Xiao Q, Zeng Y and Zhou Q: Necrolytic migratory erythema associated with glucagonoma syndrome. J Clin Oncol 28: e329-e331, 2010.

12. Remes-Troche JM, Garcia-de-Acevedo B, Zuniga-Varga J, Avila-Funes A and Orozco-Topete R: Necrolytic migratory erythema: a cutaneous clue to glucagonoma syndrome. J Eur Acad Dermatol Venereol 18: 591-595, 2004.

13. Kheir SM, Omura EF, Grizzle WE, Herrera GA and Lee I: Histologic variation in the skin lesions of the glucagonoma syndrome. Am J Surg Pathol 10: 445-453, 1986.

14. Mullans EA and Cohen PR: Iatrogenic necrolytic migratory erythema: a case report and review of nonglucagonoma-associated necrolytic migratory erythema. J Am Acad Dermatol 38: 866-873, 1998.

15. Yao JC, Eisner MP, Leary C, Dagohoy C, Phan A, Rashid A, Hassan M and Evans DB: Population-based study of islet cell carcinoma. Ann Surg Oncol 14: 3492-3500, 2007.

16. Radny P, Eigentler TK, Soennichsen K, Overkamp D, Raab HR, Viebahn R, Mueller-Horvart C, Sotlar K and Rassner G: Metastatic glucagonoma: treatment with liver transplantation. J Am Acad Dermatol 54: 344-347, 2006.

17. Poggi G, Villani L and Bernardo G: Multimodality treatment of unresectable hepatic metastases from pancreatic glucagonoma. Rare Tumors 1: e6, 2009.

18. Smith AP, Doolas A and Staren ED: Rapid resolution of necrolytic migratory erythema after glucagonoma resection. J Surg Oncol 61: 306-309, 1996.

19. Elsborg L and Glenthoj A: Effect of somatostatin in necrolytic migratory erythema of glucagonoma. Acta Med Scand 218: 245-249, 1985. 